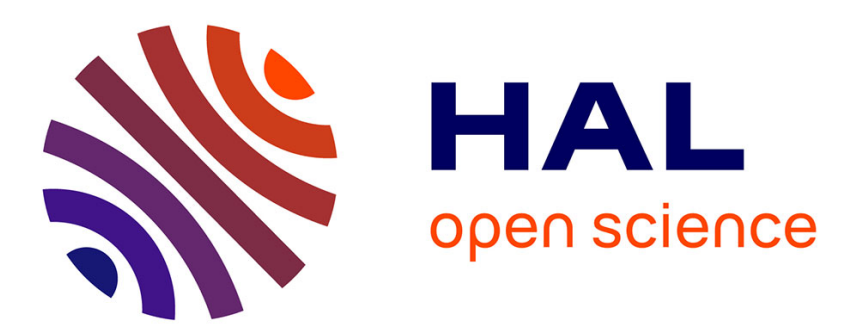

\title{
Contrôle actif du bruit par minimisation du débit volumique de structures : analyse et expériences
}

\author{
A. Berry, C. Guigou, F. Charette
}

\section{To cite this version:}

A. Berry, C. Guigou, F. Charette. Contrôle actif du bruit par minimisation du débit volumique de structures: analyse et expériences. Journal de Physique IV Proceedings, 1994, 04 (C5), pp.C5-203C5-206. 10.1051/jp4:1994539 . jpa-00253034

\section{HAL Id: jpa-00253034 https://hal.science/jpa-00253034}

Submitted on 1 Jan 1994

HAL is a multi-disciplinary open access archive for the deposit and dissemination of scientific research documents, whether they are published or not. The documents may come from teaching and research institutions in France or abroad, or from public or private research centers.
L'archive ouverte pluridisciplinaire HAL, est destinée au dépôt et à la diffusion de documents scientifiques de niveau recherche, publiés ou non, émanant des établissements d'enseignement et de recherche français ou étrangers, des laboratoires publics ou privés. 


\title{
Contrôle actif du bruit par minimisation du débit volumique de structures : analyse et expériences
}

\author{
A. BERRY, C. GUIGOU et F. CHARETTE
}

GAUS, Département de Génie Mécanique, Université de Sherbrooke, Sherbrooke, Québec J1K 2RI, Canada

The active control of structurally radiated sound requires in general a large number of error sensors on the vibrating structure. A simple control strategy, using only one error signal the volume velocity of the structure- has recently been proposed. Such a strategy allows (i) to control all the structural modes that are monopole radiators at low frequencies (i.e., with a high radiation efficiency), (ii) to keep the control set-up simple (only one information is to be minimized). The volume velocity is measured using piezoelectric PVDF strip. The shape of the PVDF strip is determined in such a way that the output signal from the PVDF sensor is directly proportional to the volume velocity induced by the structure on which it is bonded. Using a modal approach, the sensor shape is shown to depend only on the structure and sensor characteristics. However, it is independent of the excitation and the frequency. For a simply supported beam, the shape of such a PVDF sensor is shown and experimental validations are presented for the measure of the volume velocity. Active control was then implemented with this PVDF error sensor and piezoceramic actuators for different frequencies of excitation, using a one-channel feedforward adaptive LMS algorithm. Results show that sound radiation can be efficiently attenuated using this strategy when the system is driven "on-resonance". However, when the system is driven "off-resonance", the axial effects induced by the asymmetric piezoceramic actuators limit the control efficiency.

\section{INTRODUCTION}

Depuis une dizaine d'année, le contrôle actif a émergé comme une solution pratique pour réduire le rayonnement acoustique en basses fréquences [1]. Un système de contrôle actif typique requiert trois éléments: (i) des capteurs d'erreur, qui produisent un signal électrique proportionnel au signal à contrôler; (ii) un système de contrôle, qui calcule les signaux de contrôle à être appliqués à partir des signaux d'erreur; (iii) les actuateurs de contrôle, qui ré-excitent mécaniquement la structure de telle sorte que les signaux d'erreur soient minimisés.

Un des composant essentiel d'un système de contrôle actif est le capteur d'erreur. En général, pour contrôler activement le rayonnement acoustique d'une structure, des microphones sont placés dans le champ acoustique. Pour certaines applications, cette solution ne peut être implémentée. Il faut donc utiliser des capteurs vibratoires directement attachés sur la structure en vibration. Le contrôle actif du rayonnement acoustique de structures en vibration requiert généralement un nombre élevé de capteurs d'erreur sur la structure vibrante [2]. En fait, pour des structures planes, le rayonnement sonore est directement relié à la distribution vibratoire sur la surface de la structure. Il a été demontré que les modes vibratoires ayant un débit volumique non nul rayonnent plus efficacement que les autres. Une stratégie simple de contrôle actif visant à minimiser le débit volumique d'une structure a ainsi été proposée $[3,4,5]$. Cette stratégie permet donc de contrôler l'ensemble des modes vibratoires associés à une forte efficacité de rayonnement (comportement monopolaire en basses fréquences) et de conserver une architecture de contrôle simple (une seule information à minimiser). Les films piézoélectriques PVDF (polyvinylidene fluoride) ont été 
recemment utilisés comme capteurs pour le contrôle actif des vibrations et aussi du rayonnement sonore $[2,6]$. Ces films PVDF sont des capteurs étendus qui ont l'anvantage d'être très flexibles, très légers et de pouvoir être découpés pour obtenir différents signaux. Dans cette étude, la mesure du débit volumique est donc effectué au moyen d'un film PVDF dont la forme est determinée de telle sorte que le signal de ce capteur d'erreur est directement proportionnel au débit volumique de la structure. Ces formes, obtenues par décomposition sur les modes de la structure, dépendent uniquement des dimensions de la structure et de ses conditions aux limites et sont indépendantes de la fréquence et de l'excitation. Un système de contrôle actif constitué d'une poutre simplement appuyée sur laquelle se trouvent le capteur PVDF de débit volumique et deux actuateurs piézocéramiques asymétriques a été implémenté. Le contrôleur utilisé pour ces essais est de type "feedforward" harmonique basé sur un algorithme LMS adaptatif. Des résultats sont présentés et discutés pour des excitations à la résonance et hors résonance.

\section{THEORIE}

Le déplacement en flexion d'une poutre finie peut s'ecrire sous forme modale

$$
w(x)=\sum_{n=1}^{N} \hat{W}_{n} X_{n}(x),
$$

où $\hat{W}_{n}$ est l'amplitude modale du $n^{\text {ième }}$ mode, $X_{n}(x)$ est la $n^{\text {ième }}$ fonction modale associées aux conditions aux limites de la poutre, $N$ est le nombre total de mode considéré.

Le débit volumique est défini par l'intégrale sur la surface de la poutre du déplacement, c.à.d.

$$
\mathrm{D}=\mathrm{b} \int_{0}^{\mathrm{L}} \mathrm{w}(\mathrm{x}) \mathrm{dx}=\mathrm{b} \sum_{\mathrm{n}=1}^{\mathrm{N}} \hat{\mathrm{W}}_{\mathrm{n}} \int_{0}^{\mathrm{L}} \mathrm{X}_{\mathrm{n}}(\mathrm{x}) \mathrm{dx},
$$

où $b$ et $L$ sont respectivement la largeur et la longueur de la poutre.

La réponse d'un capteur PVDF dont la forme est décrite par une fonction $F(x)$ est proportionnelle à l'intégrale des contraintes sur la surface d'application du capteur, c.à.d.

$$
q=-\left(h_{b}+h_{s}\right) e_{31} \int_{0}^{L} F(x) \frac{d^{2} w}{d x^{2}} d x,
$$

où $h_{b}$ et $h_{s}$ sont respectivement l'épaisseur de la poutre et du film PVDF, et $e_{31}$ est le coefficient déformation/charge du film PVDF. On considère alors que la fonction $F(x)$ décrivant la surface couverte par le PVDF est une combinaison linéaire de la dérivée seconde des fonctions modales, c.à.d.

$$
F(x)=\sum_{i=1}^{N} \alpha_{i} \frac{d^{2} X_{i}}{d x^{2}}
$$

où les coefficients $\dot{\alpha}_{\mathrm{i}}$ sont les inconnues à déterminer. En utilisant les relations d'orthogonalité des fonctions modales, l'équation (3) se réduit à

$$
q=-\left(h_{b}+h_{s}\right) e_{31} \sum_{n=1}^{N} \hat{W}_{n} \alpha_{n} k_{n}^{4} \int_{0}^{L} X_{n}^{2}(x) d x .
$$

Il faut donc déterminer les coefficients $\alpha_{\mathrm{n}}$ de telle sorte que la réponse du capteur PVDF (équation (5)) soit proportionnelle au débit volumique (équation (2)). En identifiant chaque terme de la somme dans l'équation (5) à chaque terme de la somme dans l'équation (2), on obtient

$$
\alpha_{n}=\frac{-b}{e_{31}\left(h_{b}+h_{s}\right)} \frac{\int_{0}^{L} X_{n}(x) d x}{k_{n}^{4} \int_{0}^{L} X_{n}^{2}(x) d x} \text { pour } n=1, N .
$$

On peut alors noter que la forme du capteur PVDF est indépendente de la fréquence et de l'excitation. En effet, cette forme dépend seulement du comportement modal de la poutre ainsi que des caractéristiques de la poutre et du film PVDF. On pourra aussi observer que le coefficient $\alpha_{\mathrm{n}}$ est proportionnel à l'intégrale de

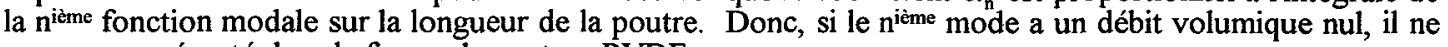
sera pas représenté dans la forme du capteur PVDF.

\section{RESULTATS EXPERIMENTAUX}

La figure 1 montre la poutre simplement supportée en aluminium qui a été utilisée dans cette partie expérimentale. Sur une face de cette poutre se trouve le capteur PVDF de débit volumique dont la forme a été obtenue à partir des équations (4) et (6). Seuls les modes impairs (modes symétriques), les plus rayonnants, sont représentés dans la fonction forme du capteur puisque ce sont les seuls à avoir un débit volumique non nul. Sur l'autre face de la poutre, deux actuateurs piézocéramiques $(2.8 \mathrm{~cm}$ de long et $1.3 \mathrm{~cm}$ 
de large) ont été collés: un pour exciter la structure et un autre pour minimiser le signal du capteur d'erreur dans le processus de contrôle. Dans cette configuration, ces actuateurs induisent à la fois de la flexion et de l'extension [7]. Les conditions d'appui simple sont assez bien verifiées expérimentalement puisque l'erreur entre les fréquences de résonance prédites par theorie et celles mesurées est inférieure à $0.6 \%$ sur les six premiers modes.

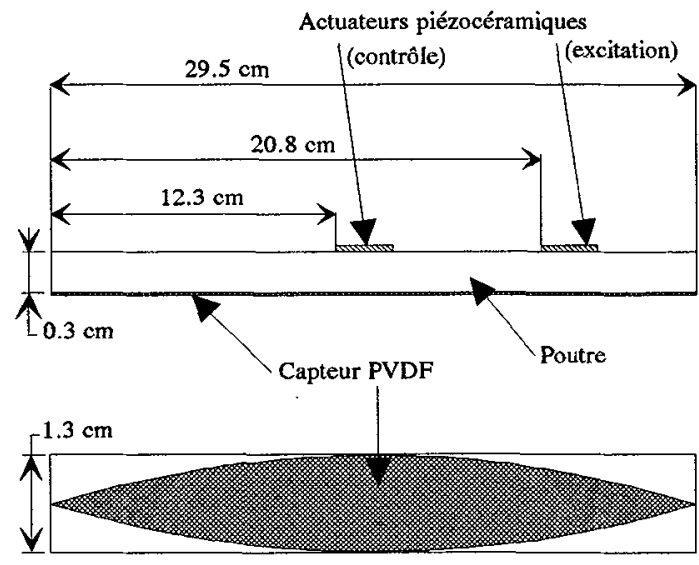

Figure 1: Montage expérimental.

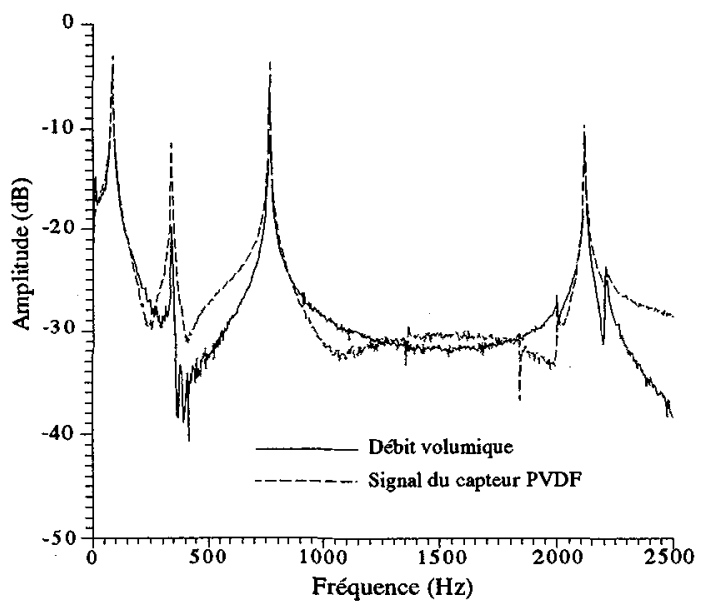

Figure 2: Comparaison du signal du PVDF et du débit volumique.

La figure 2 présente le signal du capteur PVDF et le débit volumique déterminé à partir du champ vibratoire sur la poutre. Notons que le champ vibratoire est déterminé à partir de 15 mesures le long de la poutre effectuées avec un vibromètre laser. On peut remarquer que près des résonance des modes impairs $(84,764$ et $2116 \mathrm{~Hz}$ ) les deux courbes correspondent assez bien. On pourrait s'étonner d'observer un pic à la fréquence du second mode $(340 \mathrm{~Hz})$ qui théoriquement a un débit volumique nul. Ceci est dû aux actuateurs piézocéramiques qui modifient (masse et raideur) légèrement les déformées modales. Le mode 4 n'apparait pas car l'actuateur (centré à environ 1/4 de la poutre) ne peut l'exciter. Hors résonance, les différences peuvent être attribuées au déplacement axial induit par l'actuateur [7] et qui n'est pas pris en compte dans la détermination de la forme du capteur PVDF.

Le tableau 1 montre les résultats de contrôle actif sur la poutre pour diverses fréquences d'excitation. Pour les excitations à la résonance, le contrôle est assez efficace. Il s'accompagne en général d'une diminution de la vitessse quadratique $c$ à.d du champ vibratoire, et du champ acoustique. Un cas représentatif (mode 3 ) est présenté dans les figures 3 et 4 . Il en est de même pour une fréquence proche d'une résonance de la structure $(788 \mathrm{~Hz})$. Mais pour un cas hors résonance $(552 \mathrm{~Hz})$, même si le signal du capteur PVDF est largement réduit, le débit volumique et le champ vibratoire sont en général augmentés et le champ acoustique est seulement légèrement modifié. Ceci est dû au fait que hors résonance le capteur est plus sensible aux déformations axiales induites par les actuateurs piézocéramiques que celles transversales. Les résultats d'un cas typique hors résonance $(552 \mathrm{~Hz})$ sont montrés sur les figures 5 et 6 .

Tableau 1: Résultats de contrôle actif.

\begin{tabular}{|c|c|c|c|c|}
\hline Fréquence $(\mathrm{Hz})$ & $\begin{array}{c}\text { Atténuation }(\mathrm{dB}) \\
\text { Capteur PVDF }\end{array}$ & $\begin{array}{c}\text { Atténuation }(\mathrm{dB}) \\
\text { Débit volumique }\end{array}$ & $\begin{array}{c}\text { Atténuation }(\mathrm{dB}) \\
\text { Vitesse quadratique }\end{array}$ & $\begin{array}{c}\text { Atténuation }(\mathrm{dB}) \\
\text { Champ acoustique }\end{array}$ \\
\hline 84 (mode 1$)$ & 51 & 26 & 33 & \\
\hline 340 (mode 2$)$ & 38 & 4 & 23 & 3.4 \\
\hline 552 & 34 & -5 & -0.4 & 37 \\
\hline 764 (mode 3) & 45 & 18 & 38 & 17 \\
\hline 788 & 36 & 8 & 17 & 16 \\
\hline 2116 (mode 5) & 49 & 7.5 & 16 & \\
\hline
\end{tabular}

\section{CONCLUSIONS}

Une nouvelle stratégie de contrôle actif visant à contrôler le débit volumique d'une structure a été proposée. Un nouveau type de capteur a été développé et testé pour. la mesure du débit volumique. Son 
implementation s'est montré très efficace quand la structure est excitée sur ses résonances. Pour une excitation hors résonance, l'efficacité du contrôle est limité par les effets axiaux induits par les actuateurs piézocéramiques. Ce problème peut être évité si ces effets sont pris en compte dans le design du capteur PVDF. Notons qu'un seul capteur d'erreur a permis de contrôler plusieurs modes de la structure.

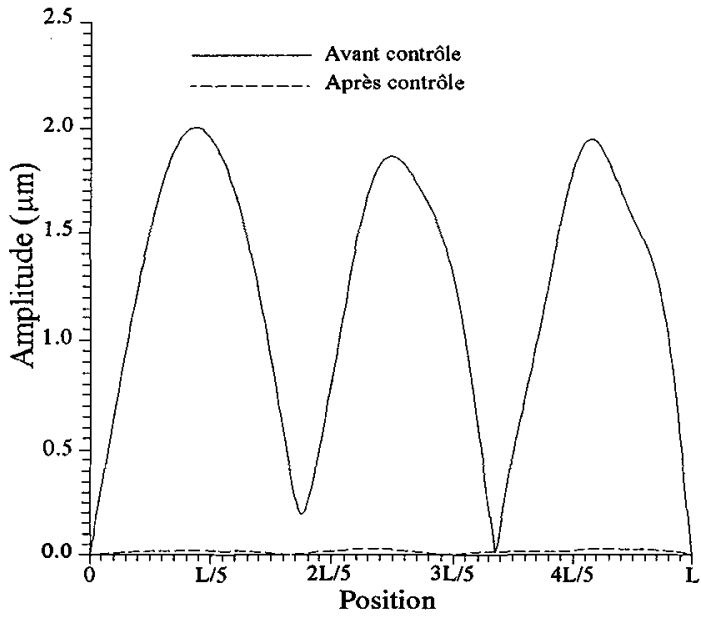

Figure 3: Déplacement à $764 \mathrm{~Hz}$ (mode 3).

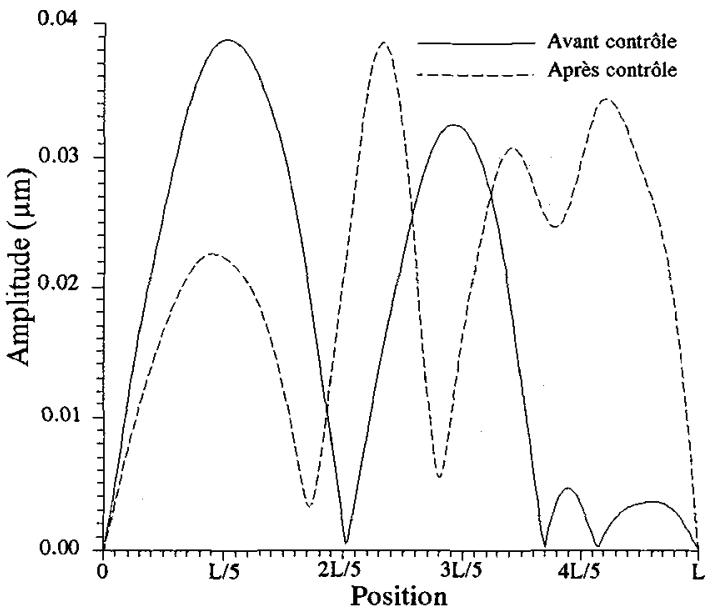

Figure 5: Déplacement à $552 \mathrm{~Hz}$ (hors résonance).

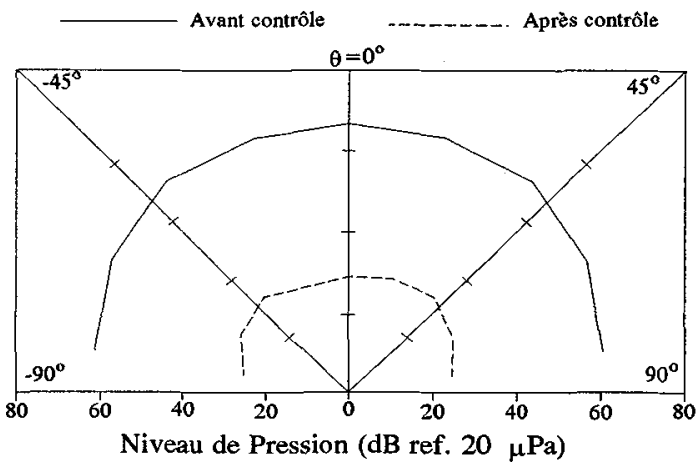

Figure 4: Champ acoustique à $764 \mathrm{~Hz}$ (mode 3).

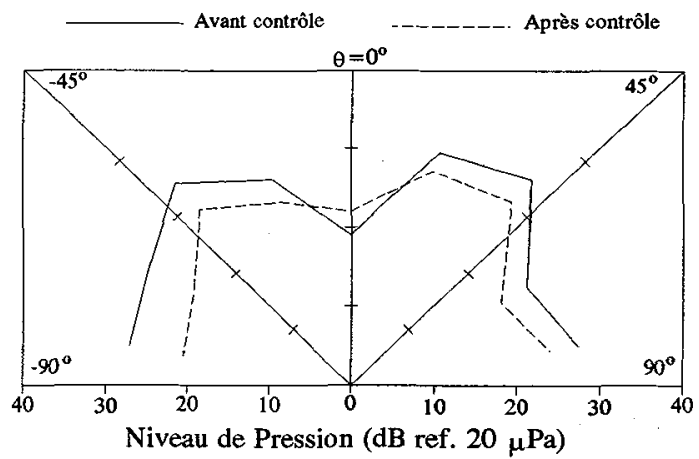

Figure 6: Champ acoustique à $552 \mathrm{~Hz}$ (hors résonance).

\section{REFERENCES}

[1] P.A. Nelson et S.J. Elliott, Active Control of Sound. (Academic Press Limited, London, 1992).

[2] R.L. Clark et C.R. Fuller, "Modal sensing of efficient acoustic radiators with PVDF distributed sensors in active structural acoustic approaches," J. Acous. Soc. Am. 91(6), 3321-3329, (1992).

[3] M.E. Johnson and S.J. Elliott, "Volume velocity sensors for active control," Proceedings of the Institute of Acoustics, Vol. 15 Part 3, 411-420, (1993).

[4] C. Guigou et A. Berry, "Active control of finite beam volume velocity using shaped PVDF sensors," J. Acous. Soc. Am. 93(4, Pt 2), 2351, (1993).

[5] C. Guigou, A. Berry et J. Nicolas, "Active control of finite beam volume velocity using shaped PVDF sensor," Soumis au J. Acous. Soc. Am., (1993).

[6] C.K. Lee et F.C. Moon, "Modal sensors/actuators," Transactions of the ASME 57, 434-441, (1990).

[7] F. Charette, C. Guigou, A. Berry et G. Plantier, "Asymetric actuation and sensing of a beam using piezoelectric materials," Soumis au J. Acous. Soc. Am., (1993). 